\title{
Novel Technique of Wire-Guided Retrograde Esophageal Dilatation in Infants Using Feeding Tubes: The "DRIFT" Technique
}

\author{
Ravi Patcharu, MS, MCh ${ }^{1}$, Karunesh Chand, MS, $M C^{1^{*}}$ and Badal Parikh, $M^{2}$ \\ ${ }^{1}$ Department of Pediatric Surgery, Army Hospital Research \& Referral, New Delhi, India \\ ${ }^{2}$ Department of Anesthesiology \& Critical Care, Army Hospital Research \& Referral, New Delhi, India
}

*Corresponding author: Dr. Karunesh Chand, MS, MCh, Department of Pediatric Surgery, Army Hospital Research \& Referral, New Delhi 110010, India, Tel: +917875545668

\begin{abstract}
Background: Esophageal dilatation is the modality of choice for managing anastomotic stricture after primary repair of esophageal atresia (EA). Balloon dilatation over guide wire is the preferred technique with minimal chances of mucosal erosion. In some cases of tight strictures, safe antegrade dilatation becomes impossible if the guidewire cannot be negotiated across the stricture. In such situations, retrograde dilatation is a safer alternative to avoid another thoracotomy for stricture resection and anastomosis. Retrograde approach to the stricture and negotiation of the guidewire requires flexible endoscopy, flouroscopy and subsequent use of dilators. We describe the 'DRIFT' (Dilatation Retrograde using Infant Feeding Tubes) technique without the use of any of these but with a safe and effective dilatation thus preserving the native esophagus.
\end{abstract}

Description of surgical technique: A neonate with anastomotic stricture following repair of EA was referred to us after a failed antegrade dilatation due to inability to negotiate the stricture. A gastrotomy was made and a cystourethroscope was used to visualise the stricture and negotiate a guidewire across it under direct vision. Retrograde dilatation was done using serially increasing sizes of feeding tubes threaded over the guidewire.

Conclusions: This simple technique of retrograde access and wire-guided dilatation is unique in that it neither requires flexible endoscopy, flouroscopy or conventional dilators.

\section{Keywords}

Esophageal atresia, Anastomotic stricture, Retrograde wire-guided dilatation, Cystourethroscope, Infant feeding tube, Failed antegrade dilatation

\begin{abstract}
Abbreviations
DRIFT: Dilatation Retrograde using Infant Feeding Tubes; EA-TEF: Esophageal Atresia with Tracheo Esophageal Fistula; EA: Esophageal Atresia; IFT: Infant Feeding Tube
\end{abstract}

\section{Introduction}

Preservation of the native esophagus is the endeavour of all surgeons managing patients of Esophageal Atresia (EA). Anastomotic strictures occur in 18-50\% of patients undergoing primary repair for EA [1] and esophageal dilatation is the modality of choice with an aim to avoid another thoractomy for stricture resection and anastomosis and failing which, eventually an esophageal replacement. The most common approach for dilatation is the antegrade method which is done using either bougies or balloon dilators under endoscopic or flouroscopic control. Based on experts' opinion, ESPGHAN-NASPGHAN Guidelines for children with EA only recommend the use of guide wire-guided dilators (bougie or balloon) [2].

In primary repair of EA, the lumen of the upper pouch is usually much wider than that of the lower esophagus and a stricture at the anastomosis does not always have its orifice leading into the centre of the lumen of the lower esophagus. Hence, a very narrow esophageal stricture though may be easily identified on an oral contrast study, but on esophagoscopy, the orifice may not be identified or may be too irregular or eccentric to be 
safely negotiated with a dilator. If attempts to pass a narrow catheter or guidewire via the esophagoscope fail, it is unsafe to attempt an endoscopic antegrade dilatation as any blind attempt increases the risk of esophageal perforation. In such cases retrograde dilatation over a transesophageal string that has been passed via a gastrostomy is much safer [3].

We describe a new technique of retrograde access across a tight stricture using a guidewire passed under direct vision, through the working channel of a cystoscope via a gastrotomy. Dilatation is performed using the 'DRIFT' (Dilatation Retrograde using Infant Feeding Tubes) technique with serially increasing sizes of infant feeding tubes (IFT) threaded over the guidewire. The result is a safe and effective 'wire-guided' dilatation without using conventional dilators, avoiding radiation exposure and without the use of flexible endocopy.

37-day-old child (weight $2.5 \mathrm{~kg}$ ) developed an anastomotic stricture after primary repair of Esophageal Atresia with distal Tracheo esophageal fistula (EA-TEF). The patient was started on antegrade dilatations but developed near complete occlusion of the lumen and a guidewire could not be negotiated across the stricture via endoscopy. As the child was severely symptomatic with inability to accept feeds and had recurrent episodes of apnea after aspirations, he was referred to the Pediatric Surgeon for a surgical intervention when the infant was 52-days-old. The infant was taken up for emergency surgery, but as the medical gastroenterologist wasn't available at that time, we undertook a novel method of retrograde access and dilatation. After making a gastrotomy, a cystourethroscope was used to access the stricture and pass a guidewire across. We also innovated a simple technique to dilate the stricture over the guidewire, which was effective, thus avoiding an esophageal replacement. The upper gastrointestinal endoscopy done one week after the retrograde dilatation showed a significant improvement in the luminal diameter as a pediatric endoscope of $6 \mathrm{~mm}$ diameter could be easily negotiated across the stricture area.

Under general anesthesia, a gastrotomy was made midway between the lesser and greater curvature. An 8.5 Fr pediatric cystourethroscope (Richard Wolf) with a $0^{\circ}$ telescope (Panoview) was connected to a camera and saline infusion was connected to the inlet port. With the surgeon standing to the right of the patient, the cystoscope was introduced into the gastrotomy. Under camera vision and manipulations of the stomach using the left hand, the cystoscope was manoeuvred cranially across the gastroesophageal junction till the area of the stricture (Figure 1). The stricture was identified and the flexible tip of a size $0.025^{\prime \prime}(0.64 \mathrm{~mm}), 150 \mathrm{~cm}$ long Niti-

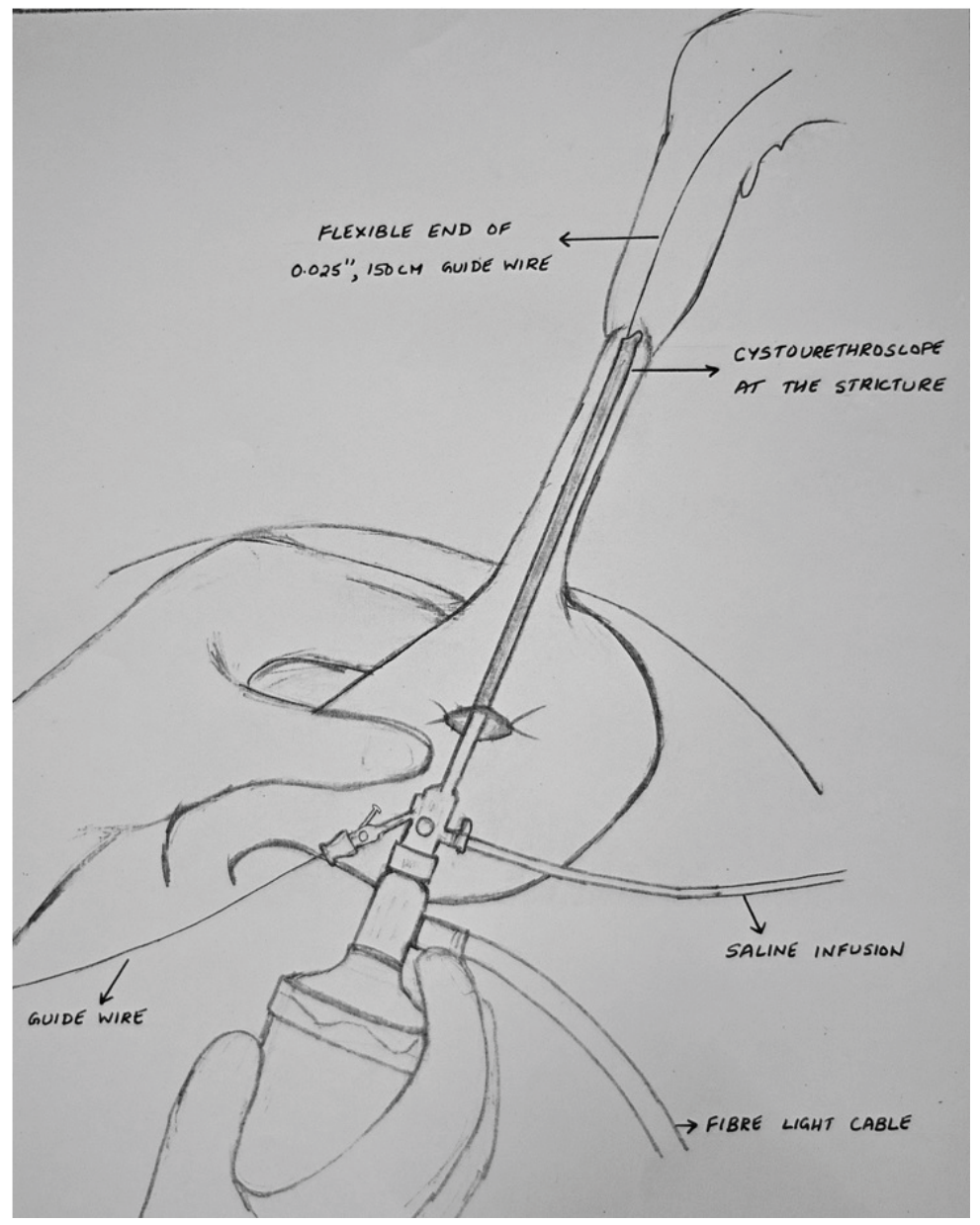

Figure 1: Technique of retrograde access to stricture using cystourethroscope. 
nol hydrophilic guidewire with $3 \mathrm{~cm}$ flexible length and straight tip was introduced into the working channel of the cystoscope and manoeuvred across the stricture into the upper esophagus (Figure 1). The anesthetist identifed the tip of the guidewire in the mouth using a laryngoscope and retrieved it using a Magill forceps and held it in a haemostat. The cystoscope was now withdrawn, leaving the guidewire in place. The inlet hub of a 5 Fr IFT was cut off and a $20 \mathrm{G} \mathrm{IV}$ cannula was introduced into its most distal eye and pierced out through the blunt tip of the IFT (Figure 2A). The needle of the cannula was removed leaving the cannula in place (Figure 2B).
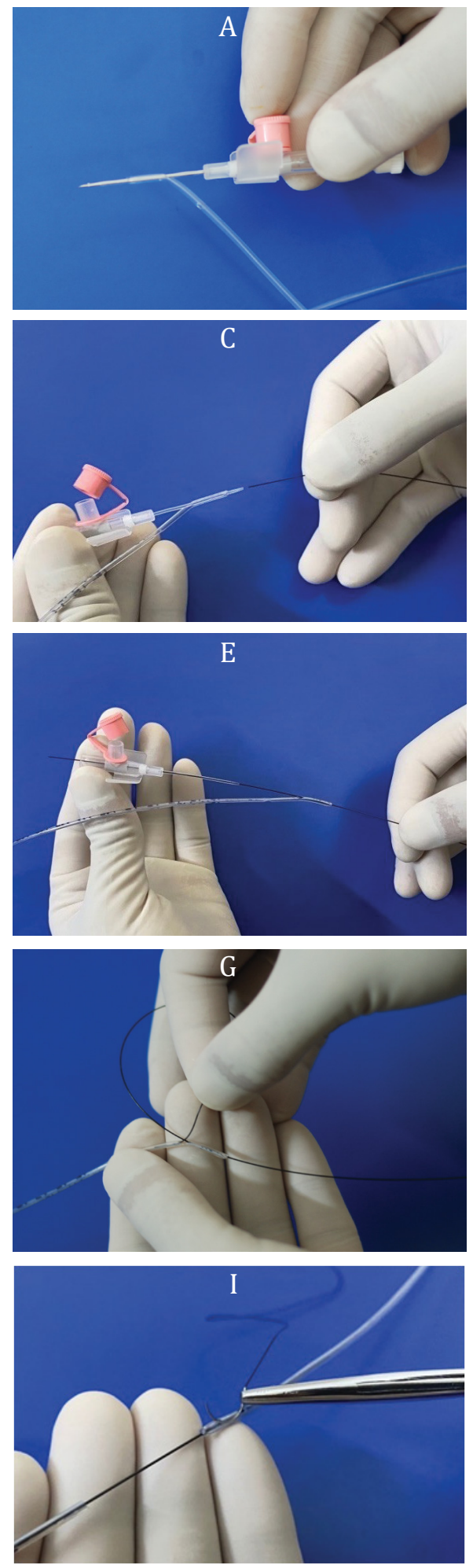
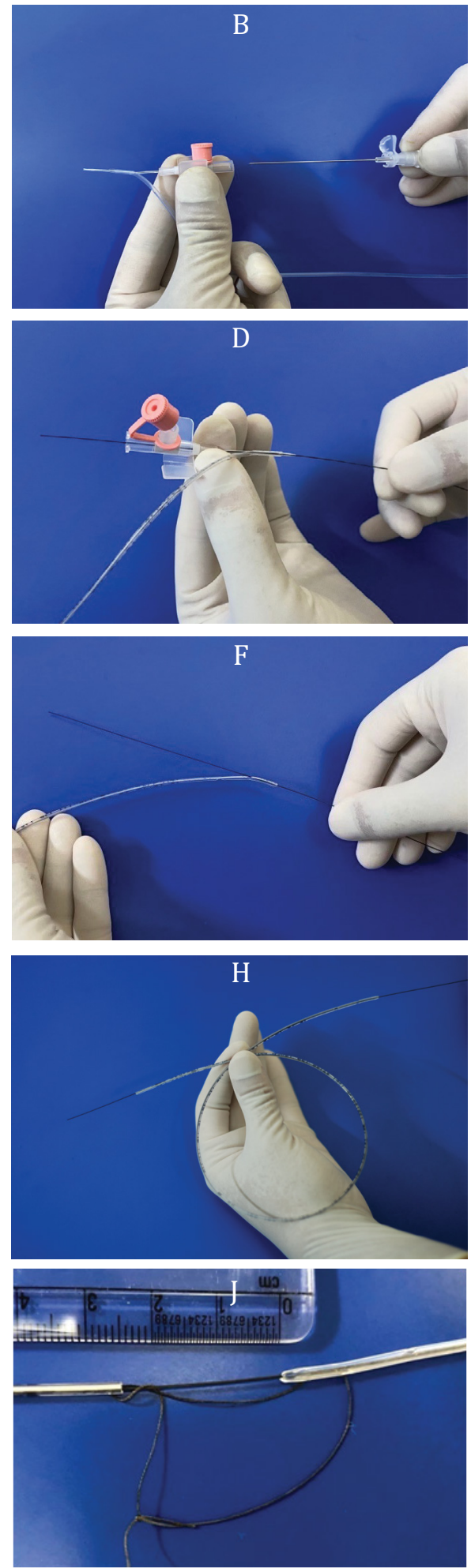

Figure 2: "DRIFT" (Dilatation Retrograde using Infant Feeding Tubes) technique image panel: A) $20 \mathrm{G}$ IV cannula is inserted into the distal opening of a $5 \mathrm{Fr}$ infant feeding tube and pierced through the blunt tip; B) Needle of the cannula is removed; C) Flexible end of 0.025 " guidewire inserted into the tip of the cannula; D) Guidewire brought out from the luer lock end of the cannula; E) IV cannula pulled out of the infant feeding tube leaving the guidewire in place; F) Guidewire tip outside the distal opening of the infant feeding tube; G) Guidewire threaded into the distal opening of the infant feeding tube; H) Guidewire brought out of the other end of the infant feeding tube; I) Bite of suture through the distal opening of 6 Fr infant feeding tube and brought out adjacent to the blunt tip; J) Silk loop between infant feeding tubes kept $2 \mathrm{~cm}$ long. 
The stomach end of the guidewire was threaded into the lumen of the cannula from its tip (Figure 2C) and brought out through its luer lock end (Figure 2D). The cannula was now pulled out of the IFT (Figure 2E and Figure 2F) and the tip of the guidewire threaded into the lumen of the IFT through the distal eye (Figure $2 \mathrm{G}$ ), till it came out of the other end of the IFT (Figure 2H). The feeding tube was now slided over the guidewire into the stomach through the gastrotomy. Lignocaine gel $1 \%$ was applied over the IFT and the end of the guidewire that was out of the mouth was pulled cranially so that the IFT could cross the strictured area of esophagus and could be seen in the mouth. Now a 6 Fr IFT was similarly threaded over the guidewire from the gastrotomy end so that the blunt tip reached the end of the $5 \mathrm{Fr}$ feeding tube. The end of the 5 Fr IFT was sutured to the tip of the 6 Fr IFT using a silk suture $2 / 0$ on a cutting needle. The bite in the 6 Fr IFT was taken through the distal eye and brought out through just adjacent to the blunt tip (Figure 2I). The loop of suture was kept about $2 \mathrm{~cm}$ long (Figure 2J). The loop acts as an additional safety feature so that the feeding tubes remain in the same track even if the guidewire dislodges by mistake. In the event of the guidewire dislodging during the procedure, the $2 \mathrm{~cm}$ loop length keeps the two serial feeding tubes slightly away during traction so that they are drawn in line. If this gap is not left and IFTs are tied too close together, the tip of the IFT tends to kink during traction.

The mouth end of the guidewire was slowly pulled cranially so that serially increasing sizes of feeding tubes could dilate the stricture (Figure 3) upto 16 Fr. A 9 Fr IFT was now introduced through one nostril and placed into the stomach antegrade. The gastrotomy was completed into a Stamms gastrostomy over a $12 \mathrm{Fr}$ Foleys catheter and the abdomen incision closed.

\section{Discussion}

Anastomotic stricture after primary repair of TEF has been defined as a narrowing at the level of the esophageal anastomosis, detected by barium contrast study and/or endoscopy, and associated with significant functional impairment and symptoms [2]. Since the first pediatric description approximately 30 years ago, esophageal dilation has become the recommended first-line treatment for anastomotic stricture following EA repair [2] with a success rate of 58-96\% [4]. There are two main categories of dilators used: Fixed-diameter pushtype dilators (bougie dilators) and radial expanding bal-

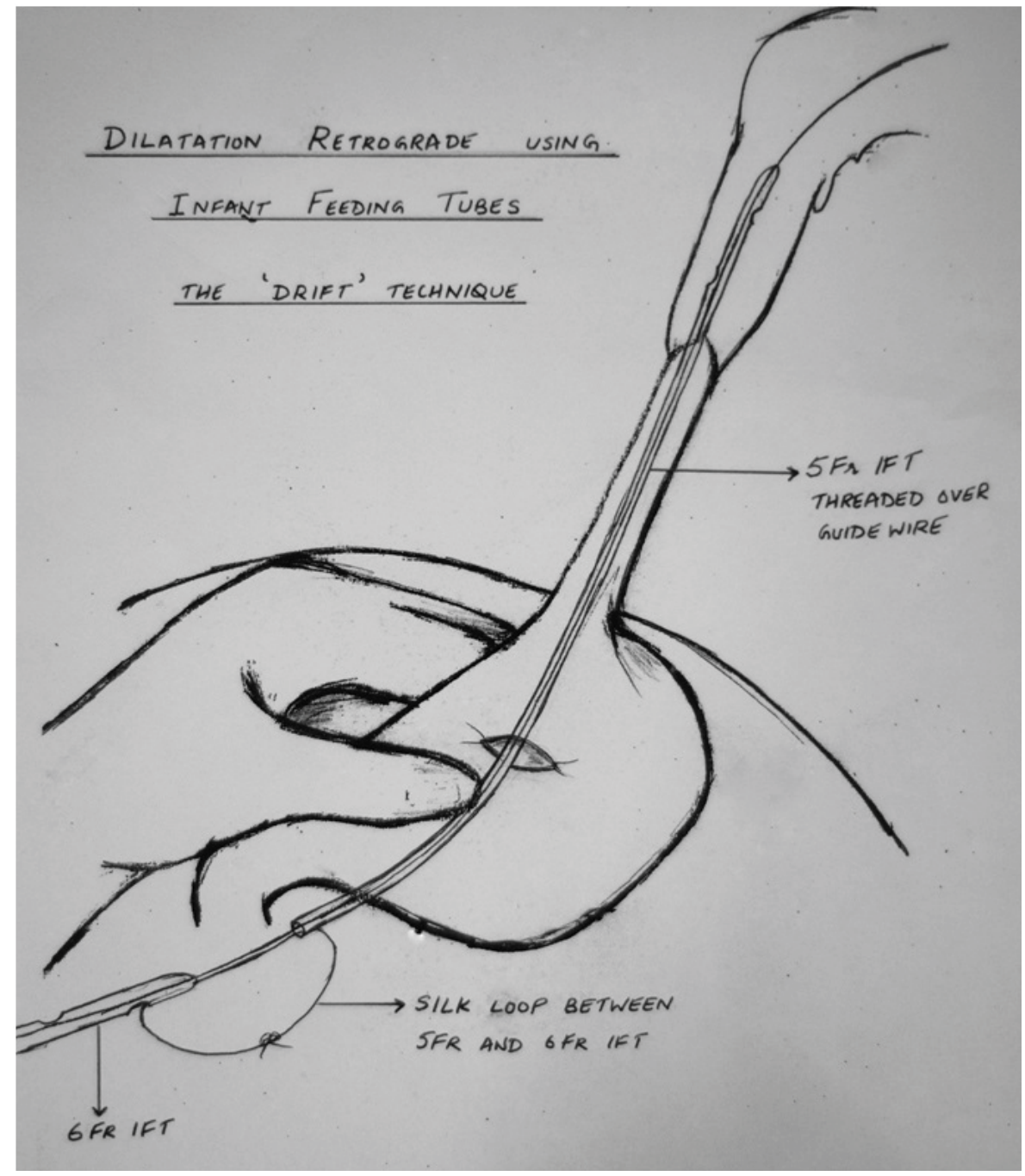

Figure 3: Dilatation Retrograde using Infant Feeding Tubes: The 'DRIFT' technique. 
Ioon dilators [5]. A wire-guided bougie or balloon dilatation assures that the dilator is following the line of the esophageal lumen, so they are generally preferred [6]. Once moderate resistance is encountered with the bougie dilator, it is generally recommended to pass no more than 3 consecutive dilators in increments of $1 \mathrm{~mm}$ in a single session for a total of $3 \mathrm{~mm}$. This consensus, known as the "rule of 3 ", is a well-established approach for mechanical dilations that is believed to improve safety and efficacy [7]. However all these dilators are expensive and hence may not be readily available in a resource challenged setup. The technique described here, provides an inexpensive and effective method of dilation.

Antegrade esophageal dilatation may fail in some cases due to the inability to pass a guidewire into the true distal lumen of the esophagus because of the distorted anatomy or because of angulation and tortuosity of the esophageal stricture [8]. In such cases, the surgical option available is retrograde dilatation via a gastrostomy. Attempts at negotiating the stricture via the distal esophagus may succeed as the distal esophagus appears to taper up towards the stricture, thus guiding the probe [3]. If this fails, then surgical exploration, resection of stricture and anastomosis needs consideration.

The concept of retrograde endoscopic dilatation approach was first described in children by Tucker in 1924 where he left an indwelling string through a gastrostomy to the esophagus that exteriorized from the nose and both ends tied together outside the body to be used as a guide for next dilatation session [9]. Theoretically the results of retrograde dilatation program are expected to be equal to antegrade approach as the only difference is the direction of approach.

As published in literature, various methods of accessing the stricture through the gastrotomy have been described like flexible endoscopes [8], sigmoidoscope [10] or through an endotracheal tube [3]. Ureteral stents or Fogarty catheters have been used to negotiate the stricture which are confirmed by flouroscopy, following which string guided dilatation was done using Tuckers dilators [3]. The disadvantage of the retrograde approach is that it needs a high level of experience in endoscopy and it takes more time than a standard antegrade approach; however, it takes much less time and has fewer complications when compared to major replacement surgery [8].

In all the retrograde approaches described in literature, the position of the guidewire needs to be confirmed using fluoroscopy, which involves radiation exposure in a child. Zamiara, et al. in their study on 78 infants analysed that due to the several radiology procedures and diagnostic studies, children with TEF are exposed to an average of $17.4 \mathrm{mSv}$ of ionizing radiation in the first 3 years of life (equivalent to 7 years of background ra- diation) [11]. Therefore rationalisation of all procedures requiring radiation exposure is warranted.

The technique described here uses a cystourethroscope to access the stricture via a gastrostomy and negotiate a guidewire across it. A pediatric cystourethroscope is commonly used by Pediatric Surgeons across the globe, due to the sheer number of urological abnormalities seen in surgical practice. In countries where medical gastroenterologists conventionally perform flexible endoscopy more commonly than Pediatric Surgeons, our technique helps in that any Pediatric Surgeon can easily do it without having to call for a medical gastroenterologist. Fluoroscopy is not required during the procedure, which has a dual advantage of avoiding radiation and also of its use in a setup where the facility of fluoroscopy is not available within the operating room.

In studies that have described a retrograde access across the stricture, the dilatation has been done using 'string-guided' dilators like Tuckers. The 'DRIFT' technique of dilatation that we describe here is 'wire-guided'. The serial dilatation begins with a 5 Fr IFT which is serially increased by $1 \mathrm{Fr}$ each time making an increase of only $0.3 \mathrm{~mm}$ each time resulting in a more gradual process of dilatation compared to the 'Rule of 3' consensus where the increments are of $1 \mathrm{~mm}$ each time. The tips of the feeding tubes are blunt and rounded making it safer to negotiate across the narrow stricture without mucosal injury. The additional string loop tied in between the feeding tubes ensures an additional safety feature to remain within the tract being dilated, even if the guidewire dislodges for any reason during the procedure.

In infants where a gastrostomy has already been made, there may be a certain level of difficulty in negotiating the cystoscope through the gastroesophageal junction and into the lower esophagus. In such cases elevating the epigastric region by placing a soft roll under the back of the patient makes it easier.

In our technique, access using a cystoscope and guidewire obviates the need of an endoscopist or endoscopy instrument, the requirement of fluoroscopy within the operating room, radiation exposure and the requirement of Tuckers dilators. It is a simple technique that has not been described before and can be replicated in any Pediatric Surgery setup and involves minimum costs. It avoids the costs involved in endoscopy, fluoroscopy and dilators and hence can be used both in resource challenged setups as well as in emergency situations.

\section{Conclusion}

Endoscopic stricture dilatation of a tight esophageal anastomotic stricture in an infant is at times challenging when guidewire access across the stricture cannot be established. Currently described approaches of ret- 
rograde access require either the expertise of flexible endoscopy or availability of flouroscopy in the operating room. The 'DRIFT' technique described here is effective, inexpensive, easily performed by Pediatric Surgeons and avoids radiation exposure to the child. It can be used safely in resource challenged setups in a safe and effective manner, thus preserving the native esophagus.

\section{Declarations of Interest}

None.

\section{Funding}

This research did not receive any specific grant from funding agencies in the public, commercial, or not-forprofit sectors.

\section{Author Contributions}

All authors have contributed equally in the study and in writing the manuscript.

\section{References}

1. Serhal L, Gottrand F, Sfeir R, Guimber D, Devos P, et al (2010) Anastomotic stricture after surgical repair of esophageal atresia: frequency, risk factors, and efficacy of esophageal bougie dilatations. J Pediatr Surg 45: 1459-1462.

2. Krishnan U, Mousa H, Dall'Oglio L, Homaira N, Rosen R, et al. (2016) ESPGHAN-NASPGHAN guidelines for the evaluation and treatment of Gastrointestinal and Nutritional complications in children with Esophageal Atresia-Tra- cheoesophageal Fistula. J Pediatr Gastroenterol Nutr 63: $550-570$.

3. Millar AJW, Rode H, Brown RA, Cywes S (1993) Negotiating the 'difficult' oesophageal stricture. Pediatr Surg Int 8: 445446.

4. Lévesque D, Baird R, Laberge J-M (2013) Refractory strictures post-esophageal atresia repair: what are the alternatives? Dis Esophagus 26: 382-387.

5. Siddiqui UD, Banerjee S, Barth B, Chauhan SS, Gottlieb $\mathrm{KT}$, et al. (2013) Tools for endoscopic stricture dilation. Gastrointest Endosc 78: 391-404.

6. Siersema PD (2008) Treatment options for esophageal strictures. Nat Clin Pract Gastroenterol Hepatol 5: 142-152.

7. Manfredi MA (2016) Endoscopic Management of Anastomotic Esophageal Strictures Secondary to Esophageal Atresia. Gastrointest Endosc Clin N Am 26: 201-219.

8. El-Asmar KM, Youssef AA (2019) Retrograde endoscopic dilatation for difficult caustic esophageal strictures: Feasibility and effectiveness. J Pediatr Surg 54: 1953-1957.

9. Tucker G (1924) LXIX Cicatricial Stenosis of the Esophagus, with Particular Reference to Treatment by Continuous String, Retrograde Bouginage with the Author's Bougie. Ann Otol Rhinol Laryngol 33: 1180-1223.

10. Kongtahworn C, Rossi NP (1972) Dilatation for severe esophageal stricture. Ann Thorac Surg 14: 678-679.

11. Zamiara P, Thomas KE, Connolly BL, Lane H, Marcon MA, et al. (2015) Long-term burden of care and radiation exposure in survivors of esophageal atresia. J Pediatr Surg 50: 1686-1690. 